\title{
Impact of Loss and Gain Forecasting on the Behavior of Pricing Decision-making
}

\author{
Inga Toma ${ }^{1, *}$, Dursun Delen ${ }^{2}$, Gregory Moscato ${ }^{1}$ \\ ${ }^{1}$ Business Administration, International University of Monaco, Principality of Monaco, Monaco \\ ${ }^{2}$ Management Science and Information Systems, Spears School of Business, Oklahoma State University, Tulsa, OK, US
}

Email address:

itoma@monaco.edu (I. Toma), dursun.delen@okstate.edu (D. Delen), gmoscato@monaco.edu (G. Moscato)

${ }^{*}$ Corresponding author

\section{To cite this article:}

Inga Toma, Dursun Delen, Gregory Moscato. Impact of Loss and Gain Forecasting on the Behavior of Pricing Decision-making. International Journal of Data Science and Analysis. Vol. 6, No. 1, 2020, pp. 12-19. doi: 10.11648/j.ijdsa.20200601.12

Received: December 29, 2019; Accepted: January 8, 2020; Published: February 4, 2020

\begin{abstract}
Recent forecasting research has shown a paradigm shift from algorithm aversion to appreciation. Despite growing trust in technological decision support, business decisions are often made based on gut feeling and intuition, ignoring part or all of the available data and information. Creating effective decision support solutions necessitates the understanding of the impact of emerging artificial intelligence and machine learning technologies on business decision-making processes. This study examines whether forecasting information delivery at a time when a business decision is made influences or changes the decision maker's mind, thereby leading to a different decision. The study employs a $2 \times 2$ between-subject experimental setting where forecasted results (gain/loss) and automated advice (risk/certainty) were crossed-examined. A sample of 137 participants was asked to make four different product price change decisions assisted by automated decision aid. The experiment involved two independent samples, one taken from Amazon Mechanical Turk workers and the other from the members of LinkedIn managerial groups. Results show that decision-makers are more likely to rely on automated recommendation and change their initial decision when forecasted decision outcomes lead to gain, whereas they would discount algorithmic aid if a loss is forecasted. This research adds to the extant literature in the field of human-technology interactions and contributes to the descriptive and prescriptive decision theories by illustrating that gain forecasting has a higher impact on the algorithm appreciation than loss forecasting.
\end{abstract}

Keywords: Profit and Loss Forecasting, Algorithms, Advice-Taking, Business Analytics, Data-Driven Decision Making, Prospect Theory

\section{Introduction}

Algorithms support managerial decision-making through forecasting and integrated artificial intelligence (AI) tools, such as virtual assistants and facial recognition applications. Despite the ubiquity of algorithms, recent human-technology interaction research has shown strong human distrust in algorithms. This traditional so-called "algorithm aversion" [1] seems to decrease and becomes to be replaced by increasing reliance [2]. Advances in AI have had profound implications in the way managers think and act. These changes of human perception of algorithms and response to their outcomes are the impetus of this research.

Most decision theories assume that all possible decision outcomes and probabilities are known. However, empirical studies in real-world decision-making contexts show a mismatch between theoretical models and managerial reality, where uncertainty is dominat [3]. Research regarding decision-makers facing risk and uncertainty presents different and often contradictory findings, concluding that decisionmaking strongly depends on the conditions under which decisions are made. According to prospect theory [4] people tend to be risk-seeking with their choices related to loss, and tend to be risk averse in their choices about gains. Assuming dominating uncertainty conditions in business, managers tend to base their decisions on gut feeling [5]. 
The question is would real-world decision-making match the principles of classical decision theories and models if AI eliminated uncertainty. Emerging AI technologies are changing business decision-making, obliterating a great deal of uncertainty conditions. For example, pricing engines, are capable of evaluating revenue streams [6], at least partially predicting decision outcomes. Nevertheless, the question remain how decision-makers will adopt these new tools and will managers change their decision-making behavior when being supported by information provided by AI tools.

Research exploring changes of business decision-maker behavior using automated advice is lacking. To fill some of that gap, the current research adds two important elements to the managerial decision-making environment: the forecast of decision outcomes and automated recommendations. Both have not received much attention in extant business decisionmaking studies. The research explores whether these two elements affect or change pricing decisions linked to negative and positive prospects. Our study delves into the roots of how decision makers behave when some uncertainty is eliminated. Decisions made based on gut feelings are also evaluated when automated advice is provided.

This research contributes to understanding the impact of automated recommendation on business decision makers' behavior when forecasted decision outcomes lead to gains and losses. The paper proposes experimental evidence based on the prospect theory background to define the impact of a computer-generated advice on human choice between positive and negative prospects. Throughout our research authors consistently find that participants appreciate computer advice when forecasted decision outcomes lead to gains and discount recommendation for choice among predicted losses.

Following this introduction, the second section undertakes a literature review of human trust in algorithms and decisionmaking under risk and uncertainty. The hypotheses on how gain and loss forecasting may affect pricing decision-making is developed in the third section. The fourth section discusses research design and the fifth section presents empirical results. The paper concludes with a discussion of findings and suggested directions for future research.

\section{Literature Review}

\subsection{Human-technology interaction}

Increasing scholarly attention has been given to humantechnology interactions, human perception of algorithms, and the forecasting accuracy of algorithms compared to human judgment. Empirical studies have shown that forecasting and recommendation systems can significantly affect individual decision making [7, 8]. However, there has been widespread discussion regarding human trust in technologies. Earlier findings from human-technology interaction papers indicate a phenomenon known as algorithm aversion: the aspect of human judgment being more important than statistical forecasting [9, 10]. Exploring the causes of algorithm aversion, research has concluded that people are concerned about algorithmic errors. They are more tolerant to bigger human mistakes than smaller algorithmic mistakes [1]. Moreover, algorithm aversion may be caused by difficulties understanding algorithmic decision-support systems [11]. Research suggests that algorithm aversion can be reduced by allowing people to modify an imperfect algorithm's forecast [12].

Notwithstanding earlier findings, more recent research has shown a true paradigm shift from algorithm aversion to algorithm appreciation. Prahl and van Swol [13] did not support this hypothesis, positing that human advice would be utilized more than computer advice. Logg et al. [2] showed that participants relied more on duplicative advice, thinking it comes from an algorithm rather than people. Researchers have highlighted the significance of machine learning, predictive algorithms, and data-prediction decision processes when solving social-science problems [14].

\subsection{Decision Making Under Risk and Uncertainty}

The early concept of uncertainty in economics, introduced by Keynes [15] and Knight [16], emphasized the difference between situations of risk and situations on uncertainty. A great deal of research on uncertainty has been conducted since then [17], but traditional classifications of conditions under which decisions are made include certainty, risk, and uncertainty [18]. For decision-making under certainty, each action is known and leads to specific outcomes. However, with risk, each action leads to one of a set of possible specific outcomes, each occurring with a known probability. With uncertainty, the consequences of each action have a set of possible specific outcomes, wherein their probabilities are completely unknown. Combinations of the three have also been considered, related to experimental evidence and statistical inferences. Recently, scholars have also introduced a concept of extreme uncertainty as unknowable conditions $[19,20]$.

The business world is considered to be in a near-constant state of uncertainty, where heuristic decision-making processes dominate [21]. Despite sufficient information and technical solutions being available to large companies, Gigerenzer [5] said that half of the time, managers secretly based their business decisions on gut feelings. Furthermore, Graham, Harvey, and Puri [22] showed that approximately half of CEOs stated their "gut feel" was an important factor affecting decision-making of capital allocation across divisions. Huang and Pearce [23] stated that decision makers facing unknowable risks used gut feelings and formal analysis together to achieve investment objectives. However, when gut feelings and formal analysis are in conflict, decision makers who are not accountable to others for their decisions will more likely rely on gut feelings than formal analyses. A comprehensive literature review on advice-taking and advice-giving [24] has shown the general tendency to dismiss advice from other sources. However, advice utilization differs, based on whether the decision task involves choice or judgment. Sanders and Manrodt [25] 
found that judgment-focused firms operated in environments characterized by higher uncertainty. This may be a factor contributing to greater reliance on subjective information.

The most influential model of making choices under risk is prospect theory, originated by Kahneman and Tversky [4, 26]. With this theory, decision outcomes are perceived as gains and losses related to a neutral reference point. This can include the status quo as an expected outcome. Outcomes that are better than reference points are gains. However, those that are worse than the reference point are considered losses. According to prospect theory, people are risk-seeking in choices between negative prospects. Thus, they prefer probable bigger losses over more certain smaller losses. However, among the corresponding positive prospects, the certainty effect contributes to a risk-averse preference, where people choose a highly likely smaller certain gain over a larger gain that is merely probable. According to the diminishing sensitivity principle of prospect theory, the marginal value of both gains and losses generally decreases with magnitude.

\section{Development of the Hypotheses}

In recent years, we have experienced significant advancements in data collection, processing, and presentation, offering the potential for greater forecast accuracy and business insights than ever before. Latest human-technology interaction studies have shown that people trust algorithms more than they do human advice. The literature on decision-making under risk implies risk aversion during choices between positive prospects and risk-seeking in choices between negative prospects. However, previous research papers lacked focus on real-time information delivery. It is still unknown whether managers would make decisions according to prospect theory if computer systems offered different recommendations at the time of decisionmaking. To fill the literature gap, authors performed inductive research with an exploratory approach. The main research question of our study was, "how do business decision makers respond to real-time business analytics advice linked to negative and positive prospects?"

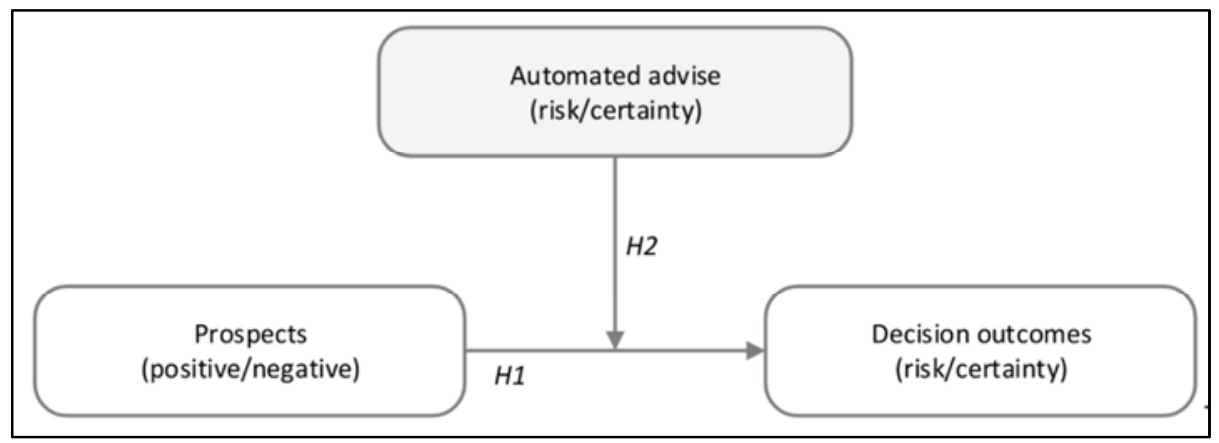

Figure 1. Theoretical Framework.

Figure 1 presents the theoretical framework of our research. Our hypotheses are built on the literature on decision-making under risk, particularly prospect theory. According to this literature, people prefer smaller certain gains over bigger but merely probable gains (risk aversion) as well as bigger but merely probable losses over smaller certain losses (risk-seeking). This leads to the following hypotheses:

H1: Prospects (i.e., gain- and loss-framed information) have an impact on decision outcomes:

$\mathrm{H} 1_{\mathrm{a}}$ : In the case of positive prospects, people prefer smaller certain gains over bigger but merely probable gains.

$\mathrm{H}_{\mathrm{b}}$ : In the case of negative prospects, people prefer bigger but merely probable losses over smaller certain losses.

$\mathrm{H} 2_{0}$ : Automated advice has no impact on the prospect- and decision-outcome relationship.

\section{Research Design}

\subsection{Participants}

Out of 242 total participants, 158 completed a study fulfilling experimental-quality requirements. The assumption was checked via the numeracy scale [27], assuming that the participants had good numeracy skills. Thus, 21 respondents who answered more than half of numeracy questions incorrect were removed, resulting in 137 participants. 34\% of respondents were female and $66 \%$ male. $62 \%$ were between the ages of 30 and $50,71 \%$ had a bachelor- or higher-level degree, 44\% came from European countries, and 39\% came from the US. The ratio of respondents engaged in business, management, information technology, and education occupations was $63 \%$.

\subsection{Task}

Authors conducted an online experiment in which the participants completed four decision-making tasks to solve a business problem. For example, should we decrease the price of one product to either boost the overall sales of the company or eliminate company's loss. In the first two tasks, the participants were asked to make a decision under uncertainty by changing the product price in a range of $-50 \%$ to $+50 \%$ of the initial price. After the decision about the new price was made, respondents received a warning with forecasted results of the decision and automated advice to adjust their new price. In case the forecasted results showed a $100 \%$ certain gain or loss, automated advice recommended changing their price to a larger, but merely probable, gain or 
loss. However, if the prediction displayed a probable gain or loss, automated advice recommended changing their price to a smaller, but certain, gain or loss. The participants had the option to agree or disagree with the advice or change the price to another value. To ensure that the respondents understood the tasks and to qualify them for the research, after making their second decision, they were asked to describe their previous tasks. The third and fourth tasks required making decisions under risk, choosing between positive and negative prospects. Instead of a price scale, respondents were asked to choose between two defined pricechange options that would result in small but certain gains or losses over merely possible gains or losses. Between decision tasks, the participants were asked to answer 11 numeracy questions to ensure they had good numeracy skills. These were necessary to ensure they understood the business problem.

\subsection{Procedure}

Participants from several LinkedIn.com groups were asked to participate in our online study to discover whether they make decisions based on intuition or reasoning or a combination of both. After reading a short introduction and answering demographic questions, the participants received a description of the first business problem, where they were asked to cut the price of a company's main product to boost overall sales. After submitting their answer, the participants were randomly split into two groups. The first group received a warning that estimated that the new price might increase the company's Return on Investment (ROI) to $30 \%$ with a $75 \%$ probability of having a $25 \%$ chance that the ROI would ultimately equal $0 \%$. The participants were advised to change their new price by $-3 \%$ to increase the company's ROI to $22.5 \%$ with a $100 \%$ certainty. The second group received a warning that estimated that the new price would increase the company's ROI to $22.5 \%$ with a $100 \%$ certainty. However, they were advised to change their new price by $-3 \%$ to increase the company's ROI to $30 \%$ with a $75 \%$ probability of a $25 \%$ chance that ROI would remain equal to $0 \%$. The participants of both groups had options to agree or disagree with the price change by $-3 \%$ or to choose another price.

In the second task, the participants were asked to change the product price to limit the expected losses of the company. After submitting their new price, the participants were randomly split into two groups, where the first group received a warning stating that new price may decrease the company's ROI to $-30 \%$ with a $75 \%$ probability having a $25 \%$ chance that ROI would remain equal to $0 \%$. The participants were advised to change their new price by $-15 \%$, thus, decreasing the company's ROI to $-22.5 \%$ with a $100 \%$ certainty. The participants of the second group received a warning, stating that the new price would decrease the company's ROI to $-22.5 \%$ with $100 \%$ certainty. The participants were advised to change their new price by $-15 \%$, thus, decreasing ROI to $-30 \%$ with a $75 \%$ probability of having a $25 \%$ chance that ROI would remain equal to $0 \%$.

In the next two tasks, the participants were asked to make a decision under risk. They were introduced to a business problem and were immediately asked to choose one of two possible options to limit the expected losses of the company. They could either change the price by $-15 \%$ to decrease company's ROI to $-30 \%$ with a $75 \%$ probability of having a $25 \%$ chance that ROI would remain equal to $0 \%$ or to change the price by $-20 \%$, thus, decreasing ROI to $-22.5 \%$ with $100 \%$ certainty. To solve the next business problem, the participants were asked to choose between two positive prospects. They could either change the price by $-15 \%$, thus, increasing company's ROI to $30 \%$ with a $75 \%$ probability of having a $25 \%$ chance that ROI would remain equal to $0 \%$, or they could change the price by $-10 \%$, thus, increasing ROI to $22.5 \%$ with $100 \%$ certainty.

\subsection{Measures and Analysis}

To test the impact of decision prospects, a $2 \times 2$ withinsubjects design was applied, where each respondent completed a task for positive and negative prospects, resulting in a complete dataset of $\mathrm{N}=274$ cases. The task of choice between gains was mapped to a positive prospect variable, whereas the task of choice between losses was mapped to a negative prospect variable. Results of both tasks were mapped to the dependent variable, "decision outcome" (i.e., risk and certainty). To measure the impact of the recommendation on the prospect and decision association, a $2 \times 2$ between-subjects design was applied, and results were analyzed separately for each prospect category, resulting in $\mathrm{N}=137$ cases. Recommendations to change the earlier chosen price were mapped to a moderator variable, "automated advice." The advice to choose $100 \%$ gain or loss was mapped to "certainty," and the advice to choose probable gain or loss was mapped to "risk." Agreement of "certainty" and disagreement of "risk" advice was mapped to the decision outcome, "certainty," whereas disagreement of "certainty," agreement of "risk," and the choice of another price was mapped to the decision outcome, "risk."

In the first analysis step, the effects of socio-demographic characteristics on the decision outcome variable were tested statistically. To examine the influence of the independent socio-demographic characteristics (e.g., gender, education, occupation) on the dependent dichotomous variable, chisquare tests were employed to identify the influence of age (metric scale) on decision outcomes. Binary logistic regression was applied. The respective findings on the effects of demographic characteristics on decision outcomes did not show a significant impact $(\mathrm{p}>0.1)$. Therefore, they were excluded from further analysis. To identify the relevant effects of prospects and automated advice on decisions, chisquare tests were again performed. In the next section, the respective findings are described.

\section{Results}

As with earlier studies $[4,26]$, results indicate that the existence of positive and negative prospects when making decisions under risk are associated with preferences to risk 
and certainty choices. With a Pearson chi-square value of 10.106 at $1 \mathrm{df}$ and $\mathrm{p}=0.001$, achieving a confidence level of more than $99 \%$, our results reject the null hypothesis, offering statistical support for the research hypothesis (H1), in which there is a significant association between prospects and decision outcomes. However, it is statistically significant, with a mean-square contingency coefficient of phi $=-0.192$ and an effective relationship size indicated as weak. In the case of positive prospects, $34 \%$ more respondents preferred smaller certain gains over bigger but merely probable gains (H1a). In the case of negative prospects, only $4 \%$ more respondents preferred bigger but merely probable losses over smaller certain losses (H1b).

Compared to results of decisions made under risk, findings of decision-making under uncertainty showed a stronger tendency. In the case of positive prospects when decisions are made under uncertainty, receiving decision outcome evaluations and recommendations to change decisions, people tended to follow the advice. With a Pearson chisquare value of 22.399 at $1 \mathrm{df}$ and $\mathrm{p}<0.001$, having a confidence level of more than $99 \%$, our results reject the null hypothesis $\left(\mathrm{H} 2_{0}\right)$, offering statistical support for the association between advice and decision outcomes. The phi coefficient of 0.404 indicates a medium effect on the association. When the participants were advised to choose a risky option over a certain one, $22 \%$ more respondents followed the advice. When they were advised to choose a certainty option over a risky one, 58\% more respondents followed the advice. In the case of negative prospects, when decisions were made under uncertainty while receiving a decision outcome evaluation and a recommendation to change the decision, a chi-square test with $p=0.240 \mathrm{did}$ not indicate a significant relationship between the advice and decision outcome. This supports the null hypothesis. With the recommendation to choose a risky loss, $50 \%$ of respondents followed the advice, and $50 \%$ did not. When recommended to choose certain loss, $40 \%$ followed the advice, whereas as $60 \%$ did not.

A comparison of positive and negative prospects (Figure 2) shows that, for negative prospects, regardless of automated advice given, the number of responses were nearly $50 \%$ for both decision outcomes: certainty or risk. For positive prospects, results show a preference of certain gain when there was no advice given and a tendency to follow the recommendation in case there was automated advice.

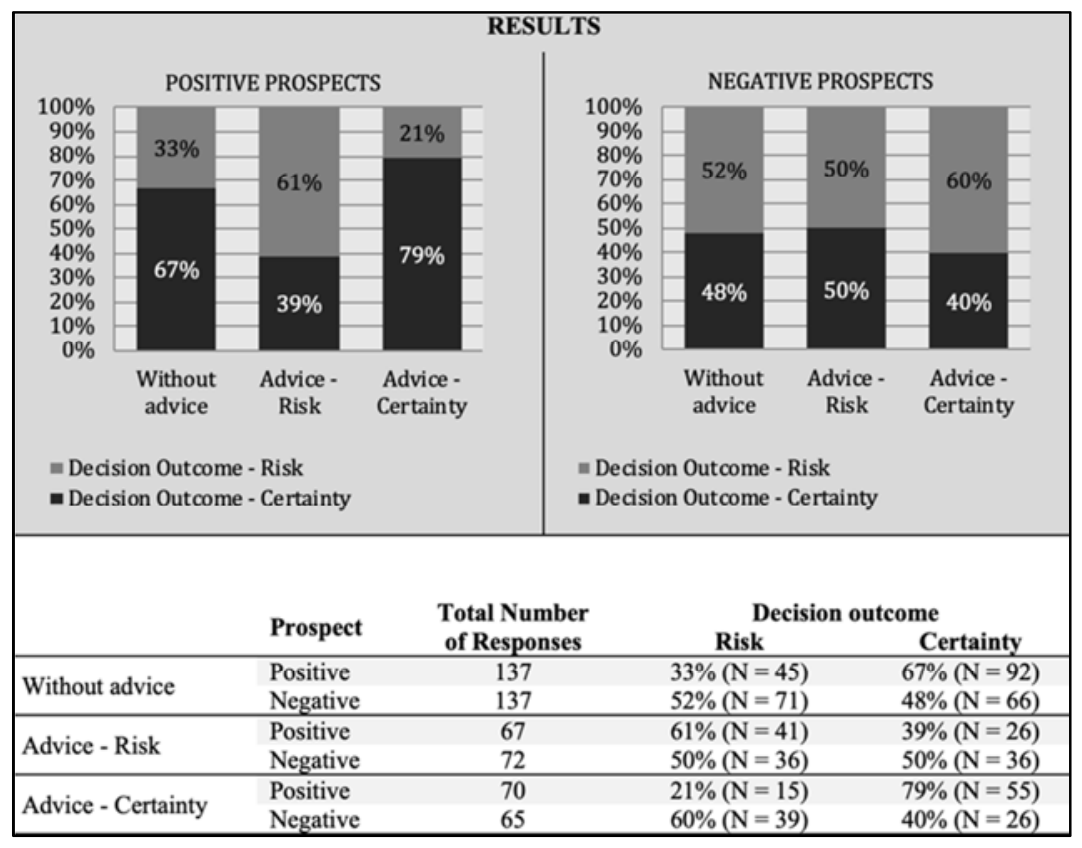

Figure 2. The Results of the Study.

When analyzing individual decision changes, authors used a two-dimensional stacked Marimekko chart, where the values of axes were based on percentages, and column heights and widths were shown relative to $100 \%$ (Figure 3 ).

On the $\mathrm{x}$-axis, displayed segments represent groups of respondents according to their preference (e.g., certainty or risk) without receiving automated advice. Considering significant differences of results for gains and losses, as seen in previous analyses, authors separated responses for positive and negative prospects. On the y-axis, column heights represent groups of respondents according to the combination of received advice and decision outcome. Highlighted areas show those groups where respondents followed the advice. However, it was opposite their initial decision preference. For example, the first column shows a group of respondents who chose certain gain, and the highlighted segment in the first column represents respondents who followed the advice to choose risky gains, thus, allowing a recommendation to influence their decision. Similarly, the second column shows a group of respondents who have chosen a risky gain when no advice was given, and the highlighted area represents a group of people who followed the advice to choose a certain gain. 


\section{DECISION CHANGES}

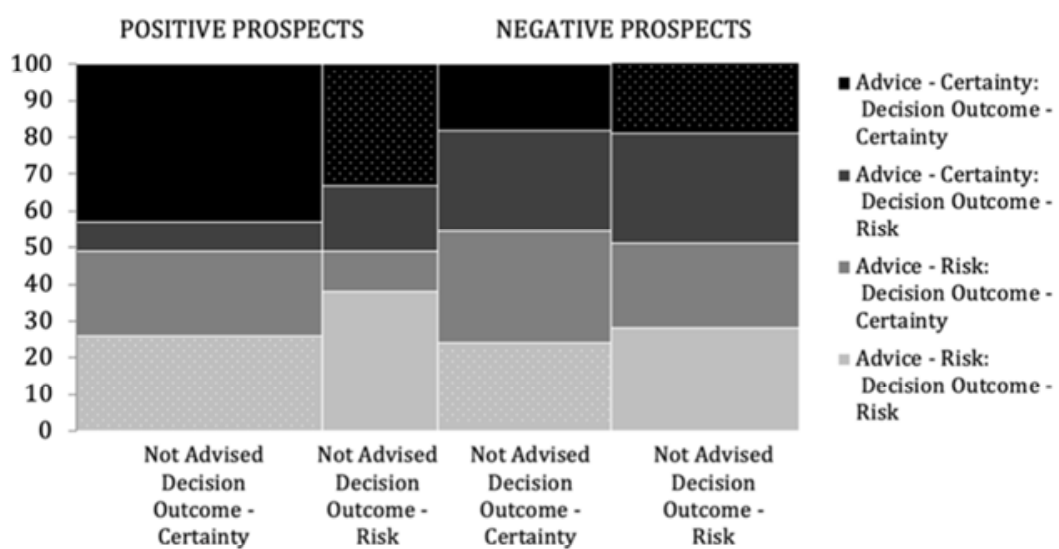

Advice influenced preference change

\begin{tabular}{clc}
\hline & \multicolumn{2}{c}{ Prospect } \\
\hline Not Advised Decision Outcome - Certainty & Positive & Negative \\
\hline Advice - Certainty & $\mathrm{N}=\mathbf{9 2}$ & $\mathrm{N}=\mathbf{6 6}$ \\
Advised Decision Outcome - Certainty & $\mathrm{N}=47$ & $\mathrm{~N}=30$ \\
Advised Decision Outcome - Risk & $\mathrm{N}=40$ & $\mathrm{~N}=12$ \\
Advice - Risk & $\mathrm{N}=7$ & $\mathrm{~N}=18$ \\
Advised Decision Outcome - Certainty & $\mathrm{N}=45$ & $\mathrm{~N}=36$ \\
Advised Decision Outcome - Risk & $\mathrm{N}=21$ & $\mathrm{~N}=20$ \\
\hline Not Advised Decision Outcome - Risk & $\mathrm{N}=24$ & $\mathrm{~N}=16$ \\
\hline Advice - Certainty & $\mathrm{N}=\mathbf{4 5}$ & $\mathrm{N}=\mathbf{7 1}$ \\
Advised Decision Outcome - Certainty & $\mathrm{N}=23$ & $\mathrm{~N}=35$ \\
Advised Decision Outcome - Risk & $\mathrm{N}=15$ & $\mathrm{~N}=14$ \\
Advice - Risk & $\mathrm{N}=8$ & $\mathrm{~N}=21$ \\
Advised Decision Outcome - Certainty & $\mathrm{N}=22$ & $\mathrm{~N}=36$ \\
Advised Decision Outcome - Risk & $\mathrm{N}=5$ & $\mathrm{~N}=16$ \\
\hline
\end{tabular}

Figure 3. Illustration of the Decision Changes.

Findings show that, out of all respondents preferring certainty over risk between positive prospects, after receiving advice to choose the risky choice, $53 \%$ of the participants agreed to the change, whereas in the same situation, between negative prospects, $44 \%$ of respondents agreed. Likewise, out of those who preferred the risky option over certainty between positive prospects, after receiving advice to choose the certain choice, $65 \%$ agreed to the change, whereas, in the same situation between negative prospects, $40 \%$ agreed to the change.

\section{Discussion}

Our empirical research supports the prospect theory, showing that, with decision making under risk, people tend to be risk averse when choosing between positive prospects, and they tend to be risk-seeking when choosing between negative prospects. However, research findings show that automated advice has had a significant impact on business decisions regarding companies' gains, whereas it has had a low impact on decisions regarding losses.

Yeomans et al. [11] stated that recommendations must be accurate and understood to reduce aversion to algorithms. In our experiments, predictions were fictitious, and the recommendation for gains and losses were displayed using the same format, altering only the probabilities and prospects. This ensured that the understanding of the recommendations for gains and losses would be identical. Notwithstanding, results showed a significant impact of recommendations on decisions about gains and a low impact on decisions about losses. As recommendation accuracy very often could not be evaluated before decisions were made, authors assume that 
this had a low impact on decisions.

Our work suggests further that people judge a recommender system more by how it fits their belief system rather than what or how it recommends. Analyzing decisions on an individual basis, research results show that, on average, in $50 \%$ of cases, the participants who received a recommendation different than their original decision preference still followed the advice, whereas the other $50 \%$ stayed with their initial choice, thereby supporting Gigerenzer's findings about managers who, in $50 \%$ of cases, based their business decisions on gut feelings, ignoring available data [5]. Research findings have shown also that people were more flexible when following recommendations about positive prospects than negative ones.

\section{Conclusion}

Authors performed inductive research with an exploratory approach looking for answers on the main research question"how do business decision makers respond to real-time business analytics advice linked to negative and positive prospects". Results indicate that the existence of positive and negative prospects when making decisions under risk are associated with preferences to risk and certainty choices. There is a significant association between prospects and decision outcomes. Respondents preferred bigger but merely probable losses over smaller certain losses. Results did not indicate a significant relationship between the advice and decision outcome.

Given that automated advice has a higher impact on decisions about gains, authors recommend the integration of such recommendations into analytical business solutions. However, in situations where decisions should be made to eliminate loss, authors recommend providing information without giving automated advice, because there is a high chance of making decisions based on gut feelings and ignoring the provided advice. Considering the significant association between automated advice and decision outcomes, authors propose that AI be used to impact decision-making and predict decision-maker behavior. This will be an interesting future research using machine-learning techniques. Future human-technology interaction research should further investigate domains where people trust and rely on algorithms. An interesting avenue for future research involves exploring the cause of current paradigm shifts, from discounting algorithms to relying on them. An examination of changes in human behavior interacting with technology across time may also significantly affect future technology development.

\section{References}

[1] B. J. Dietvorst, J. P. Simmons, and C. Massey, "Algorithm Aversion: People Erroneously Avoid Algorithms After Seeing Them Err" in Journal of Experimental Psychology: General, vol. 144 no. 1,2015 , pp. 114-126.
[2] J. M. Logg, J. A. Minson, and D. A. Moore, "Algorithm Appreciation: People Prefer Algorithmic To Human Judgment" in Harvard Business School NOM Unit Working $\begin{array}{llll}\text { Paper no. } & \text { no17-086. }\end{array}$ http://dx.doi.org/10.2139/ssrn.2941774.

[3] F. Artinger, M. Petersen, G. Gigerenzer, and J. Weibler, "Heuristics as adaptive decision strategies in management", in Journal of Organizational Behavior, vol. 36, 2015, pp. 33-52. http://doi.org/10.1002/job.1950.

[4] D. Kahneman and A. Tversky, "Prospect Theory: An Analysis of Decision under Risk" in Econometrica, vol. 47 no. 2, 1979, pp. 263-291.

[5] G. Gigerenzer, Risk Savy - How to Make Good Decisions, London: Penguin Books Ltd, 2015.

[6] A. Gajewar, J. Yang, D. Chen, and P. Chen, "Patent Application Publication Pricing Engine Revenue Evaluation", United States, 2013, available at: https://patentimages.storage.googleapis.com/17/61/e8/012aff2 c6dd394/US20130290069A1.pdf (accessed 16 September 2019).

[7] D. Waddell and A. S. Sohal, "Forecasting: The Key to Managerial Decision Making" in Management Decision, vol. 32 no. 1, 1994, pp. 41-49.

[8] P. De, Y. Hu, and M. S. Rahman, "Technology Usage and Online Sales: An Empirical Study" in Management Science, vol. 56 no. 11, 2010, pp. 1930-1945.

[9] Dzindolet, M. T., Pierce, L. G., Beck, H. P. and Dawe, L. A. (2002), "The Perceived Utility of Human and Automated Aids in a Visual Detection Task", Human Factors, Vol. 44 No. 1, pp. 79-94. http://dx.doi.org/10.1518/0018720024494856.

[10] Fildes, R. and Goodwin, P. (2007), "Against Your Better Judgment? How Organizations Can Improve Their Use of Management Judgment in Forecasting", INFORMS Journal on Applied Analytics, Vol. 37 No. 6, pp. 570-576. https://doi.org/10.1287/inte.1070.0309.

[11] Yeomans, M., Shah, A., Mullainathan, S. and Kleinberg, J. (2019), "Making Sense of Recommendations", Journal of Behavioral Decision Making, Vol. 32 No. 4, pp. 403-414 https://doi.org/10.1002/bdm.2118.

[12] Dietvorst, B. J., Simmons, J. P. and Massey, C. (2016), "Overcoming Algorithm Aversion: People will Use Imperfect Algorithms If They Can (Even Slightly) Modify Them", Management Science, Vol. 64 No. 3, pp. 1155-1170. http://dx.doi.org/10.1287/mnsc.2016.2643.

[13] Prahl, A. and Van Swol, L. (2017), "Understanding algorithm aversion: When is advice from automation discounted?", Journal of Forecasting, Vol. 36 No. 6, pp. 691-702. http://doi.org/10.1002/for.2464.

[14] Kleinberg, J., Lakkaraju, H., Leskovec, J., Ludwig, J. and Mullainathan, S. (2018), "Human Decisions and Machine Predictions", The Quarterly Journal of Economics, Vol. 133 No. 1, pp. 237-293. http://doi.org/10.1093/qje/qjx032.

[15] Keynes, J. M. (1921), A treatise on probability, Macmillan and Co., London, England.

[16] Knight, F. H. (1921), Risk, Uncertainty and Profit, Houghton Mifflin, Boston. 
[17] Faulkner, P., Feduzi, A. and Runde, J. (2017), "Unknowns, Black Swans and the risk/uncertainty distinction", Cambridge Journal of Economics, Vol. 41 No. 5, pp. 1279-1302. https://doi.org/10.1093/cje/bex035.

[18] Luce, R. D. and Raiffa, H. (1957), Games and decisions: Introduction and critical survey, Wiley, Oxford, England.

[19] Gomory, R. (1995), "The Known, the Unknown and the Unknowable", Scientific American, Vol. 272 No. 6, pp. 120. http://www.jstor.org/stable/24980843.

[20] Diebold, F. X., Doherty, N. A. and Herring, R. J. (2010), The Known, the Unknown, and the Unknowable in Financial Risk Management, Princeton University Press.

[21] Mousavi, S. and Gigerenzer, G. (2014), "Risk, uncertainty, and heuristics", Journal of Business Research, Vol. 67 No. 8, pp. 1671-1678. http://doi.org/10.1016/j.jbusres.2014.02.013.

[22] Graham, J. R., Harvey, C. R. and Puri, M. (2015), "Capital allocation and delegation of decision-making authority within firms", Journal of Financial Economics, Vol. 115 No. 3, pp. 449-470 http://doi.org/10.1016/j.jfineco.2014.10.011.

[23] Huang, L. and Pearce, J. L. (2015), "Managing the
Unknowable: The Effectiveness of Early-stage Investor Gut Feel in Entrepreneurial Investment Decisions", Administrative Science Quarterly, Vol. 60 No. 4, pp. 634-670. http://doi.org/10.1177/0001839215597270.

[24] Bonaccio, S. and Dalal, R. S. (2006), "Advice taking and decision-making: An integrative literature review, and implications for the organizational sciences", Organizational Behavior and Human Decision Processes, Vol. 101 No. 2, pp. 127-151. http://doi.org/10.1016/j.obhdp.2006.07.001.

[25] Sanders, N. R. and Manrodt, K. B. (2003), "The efficacy of using judgmental versus quantitative forecasting methods in practice", Omega, Vol. 31 No. 6, pp. 511-522. http://doi.org/10.1016/j.omega.2003.08.007.

[26] Tversky, A. and Kahneman, D. (1992), "Advances in Prospect Theory: Cumulative Representation of Uncertainty", Journal of Risk and Uncertainty, Vol. 5 No. 4, pp. 297-323. https://doi.org/10.1007/BF00122574.

[27] Lipkus, I. M., Samsa, G. and Rimer, B. K. (2001), "General Performance on a Numeracy Scale among Highly Educated Samples", Medical Decision Making, Vol. 21 No. 1, pp. 3744. https://doi.org/10.1177/0272989X0102100105. 